\title{
Thalamus gates active dendritic computations in cortex during sensory processing
}

\author{
Jason M. Guest ${ }^{1 \#} \uparrow$, Arco Bast ${ }^{1 \#}$, Rajeevan T. Narayanan ${ }^{1} \ddagger$, Marcel Oberlaender ${ }^{1 *}$ \\ ${ }^{1}$ Max Planck Group: In Silico Brain Sciences, Center of Advanced European Studies and Research \\ (caesar), Ludwig Erhard Allee 2, 53175 Bonn, Germany. \\ ${ }^{\#}$ These authors contributed equally to this work. \\ $\dagger$ current address: Max Planck Florida Institute for Neuroscience, Jupiter, FL, USA. \\ tcurrent address: University of Oxford, Oxford, UK. \\ *Corresponding author. Email: marcel.oberlaender@caesar.de
}

Perception is causally linked to a calcium-dependent spiking mechanism that is built into the distal dendrites of layer 5 pyramidal tract neurons - the major output cell type of the cerebral cortex. It is yet unclear which circuits activate this cellular mechanism upon sensory stimulation. Here we found that the same thalamocortical axons that relay sensory signals to layer 4 also densely target the dendritic domain by which pyramidal tract neurons initiate calcium spikes. Distal dendritic inputs, which normally appear greatly attenuated at the cell body, thereby generate bursts of action potentials in cortical output during sensory processing. Our findings indicate that thalamus gates an active dendritic mechanism to facilitate the combination of sensory signals with top-down information streams into cortical output. Thus, in addition to being the central hub for sensory signals, thalamus is also likely to ensure that the signals it relays to cortex are perceived by the animal.

\section{Introduction}

The ability to combine sensory signals with internal information streams is a hallmark feature of the cerebral cortex, forming the basis for perception and cognition, and the resulting behavior. It is thought that the pyramidal tract neurons in cortical layer 5 (L5PTs) are key for such combination processes $(1,2)$. Along their extensive dendrites, these major cortical output neurons combine information streams arriving at all layers, and then broadcast the results of this combination to a variety of downstream subcortical brain regions (3). In order to contribute to cortical output, distal dendritic inputs must, however, cross a high threshold to evoke dendritic calcium action potentials (APs) (4), but can then generate bursts of axonal action potentials (5). Direct evidence that this calcium-dependent spiking mechanism is utilized to implement higher brain functions was recently reported for the L5PTs in the vibrissa-related part of mouse primary somatosensory cortex (vS1) - the barrel cortex (2). Elevated calcium activity in the distal dendrites, in combination with burst firing, correlated with the threshold for perceiving whisker stimuli (6). Manipulations of the dendritic calcium domain even allowed to lower or raise the perceptual threshold. The circuits underlying these remarkable observations have yet to be revealed. This is particularly challenging, as it remains still unclear which circuits underlie the sensory-evoked responses of L5PTs in general. 
In conventional views of the cortical circuitry, L5PTs are considered as terminal points for sensory processing, assuming that sensory input is transformed as excitation spreads along connections from thalamus to layer 4 , then to layers $2 / 3$, to layers $5 / 6$, and finally to downstream subcortical regions (7-9). However, inactivating layer 4 was shown to have no effect on the sensory-evoked responses of L5PTs (10), whose onset latencies generally rival or even precede those in layer 4 (10-14). It was therefore suggested that sensory-evoked responses in the major cortical output neurons might be driven directly by the thalamus (10). However, we recently reported that inactivating a specific subpopulation of neurons in layer 6 abolished sensory-evoked APs in the L5PTs (13). Thus, synaptic input from the thalamus is generally not sufficient to drive cortical output directly. Therefore, here we asked: What is the actual relevance of thalamocortical input to L5PTs, and could this direct source of sensory information to the major cortical output neurons contribute to their role during perception?

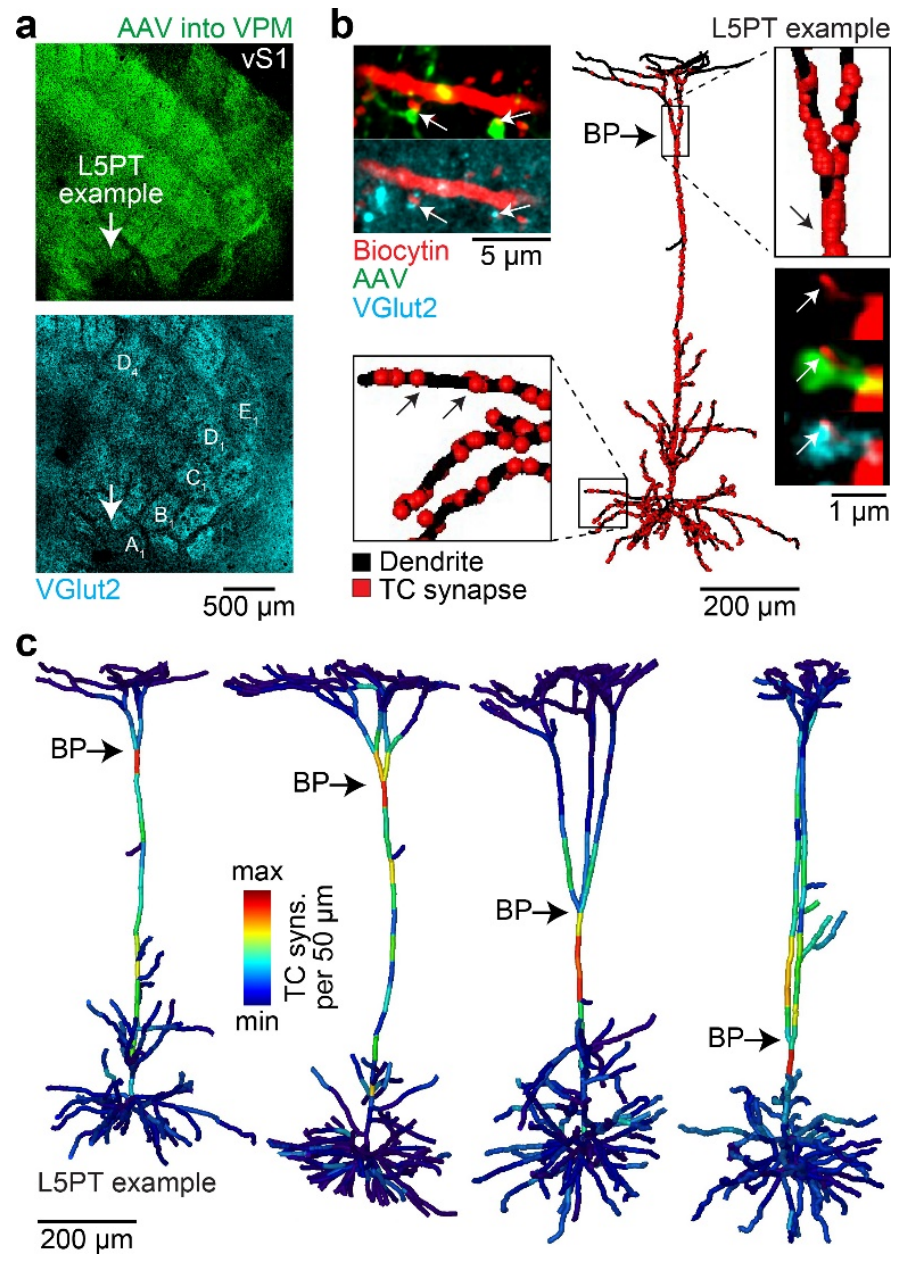

Fig. 1: Identifying thalamocortical synapses along the dendrites of in vivo recorded neurons. a. Example of recorded and reconstructed pyramidal tract neuron in layer 5 (L5PT) of the vibrissa-related part of rat primary somatosensory cortex (vS1). Top: image of layer 4 in vS1 shows axons infected by an adeno-associated virus (AAV) injected into the ventral posterior medial thalamic nucleus (VPM; Fig. S1a). Bottom: image shows the expression of the vesicular glutamate transporter 2 (VGlut2) - a marker for primary thalamocortical (TC) synapses. The arrows denote the location of the apical dendrite of a biocytin labeled L5PT (i.e., the cell body is located in a deeper histological section). b. Reconstruction of the L5PT from panel a. Arrows in zoom-ins denote the TC synapse examples in the super-resolution microscopy images. c. Density of TC synapses per $50 \mu \mathrm{m}$ dendritic length for four L5PT examples (including the one from panels $\mathrm{a} / \mathrm{b}$ ). The highest density of TC synapses correlates with the location of the primary branch point (BP) of the apical dendrite in all reconstructed L5PTs ( $n=10$; Fig. S2b).

To address these questions quantitatively, we reconstructed the distributions of thalamocortical (TC) synapses along the dendrites of in vivo recorded neurons in rat barrel cortex. For this purpose, we injected an adeno-associated virus (AAV-syn-hChr2-mCherry) into the primary thalamus of the whisker system (Fig. S1a/b) - the ventral posterior medial nucleus (VPM). We performed cell-attached recordings in the barrel cortex of virus injected rats, and in vivo labeled the recorded neurons with biocytin for post-hoc reconstruction of the virus-infected TC synapses along their dendrites. We converted the reconstructions into biophysically detailed multi-compartmental models that captured the complex intrinsic physiology of 
L5PTs - including calcium APs and burst firing. We combined these cellular models with a recently reported circuit model for the local intracortical (IC) connectivity of the rat barrel cortex (15). By performing simulations of sensory-evoked signal flow through this multi-scale model (13), we then explored in silico how active dendritic integration of anatomically and functionally constrained TC and IC inputs could contribute to the recorded cortical output patterns. Finally, we optogenetically manipulated the TC synapses, while recording from the neurons that were used for the simulations. These experiments provided direct empirical evidence for the in silico predicted function of the TC $\rightarrow$ L5PT pathway demonstrating in vivo how manipulations of TC circuits can lead to the transition of cortical output patterns from single AP to burst firing.

\section{Results}

We reconstructed the locations of 13,296 TC synapses along the dendrites of 10 in vivo labeled L5PTs in the barrel cortex from 9 virus injected rats (Fig. 1a). The TC synapses were identified via super-resolution light microscopy as contact sites between dendritic spines and virus-infected axonal boutons (Fig. 1b) that co-expressed the vesicular glutamate transporter 2 (VGlut2) - a specific marker for primary TC synapses (Fig. S1c/d). Surprisingly, for each L5PT we observed that TC synapses accumulate most densely within a region of the apical dendrite that is proximal to its primary branch point (Fig. 1c). This accumulation did not reflect higher spine densities in this region of the apical dendrite (Fig. S2a), and it was observed irrespective of whether the primary branch point was located in layers 2/3, layer 4 or layer 5 (Fig. S2b/c). This wiring specificity was not apparent in other excitatory cell types (Fig. S2d). Thus, although axons from the primary thalamus innervate all dendritic domains of deep layer neurons (Fig. S2e), we found that they specifically target the apical dendrites of L5PTs around their primary branch points, where TC synapses are hence four times more abundant compared to other parts of the dendrite (Fig. S2a).

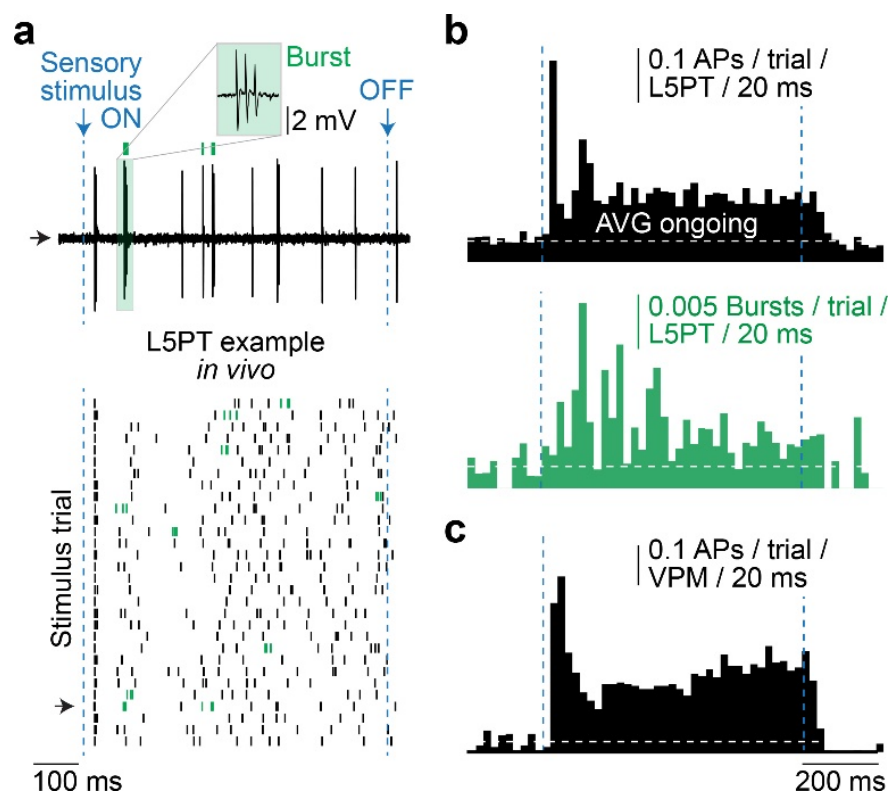

Fig. 2: Cortical output neurons show increased burst firing throughout sensory stimulation. a. Example recording of the L5PT shown in Fig. $1 \mathbf{b}$. The trace in the top panel shows action potentials (APs) recorded during one example trial in which facial whiskers were deflected for $700 \mathrm{~ms}$ (Methods). We defined bursts as three (or more) APs that occurred within $30 \mathrm{~ms}$, as such events are likely to reflect calcium APs (5). The bottom panel shows APs recorded across 30 stimulus trials (the arrow denotes the example trial). b. Post-stimulus time histograms (PSTHs) of APs (top) and bursts (bottom) across L5PTs $(n=25)$. The white dashed lines represent ongoing activity preceding sensory stimuli. PSTHs for other excitatory cell types and for inhibitory neurons are shown in Fig. S3. c. PSTH across relay cells $(n=8)$ in VPM thalamus.

What could be the functional relevance of this remarkable wiring specificity? The region around the primary branch point of the apical dendrite reflects the initiation zone for calcium APs (4, 16-19). Dense innervation of this domain by TC synapses could thereby facilitate the generation of calcium APs upon sensory stimulation, which might result in increased burst firing by L5PTs, but not in other cell types that 
lack this wiring specificity. To test this possibility, we analyzed the AP activity in response to whisker stimulations (Methods) for 90 neurons across layers 2 to 6 of the barrel cortex from 37 rats (Fig. 2a), representing both excitatory and inhibitory cell types. For all morphologically identified L5PTs ( $\mathrm{n}=25$ including the 10 with reconstructed TC synapses) we observed increased burst rates during sensory stimulation (Fig. 2b). Although bursts were observed for neurons across layers 2 to 6, among neurons of different excitatory cell types $(n=59)$, burst rates that remained elevated for the duration of the stimulus were observed exclusively in L5PTs (Fig. S3a). Inhibitory neurons $(n=31)$ also showed increased burst rates throughout the stimulus duration, but in contrast to L5PTs, they elicited bursts reliably at stimulus onsets (Fig. S3b). Thus, consistent with the anatomy, and with recent reports in behaving animals (2, 20), we found that increased burst firing during sensory processing appears to be a neurophysiological signature that distinguishes L5PTs from other excitatory cell types in barrel cortex.

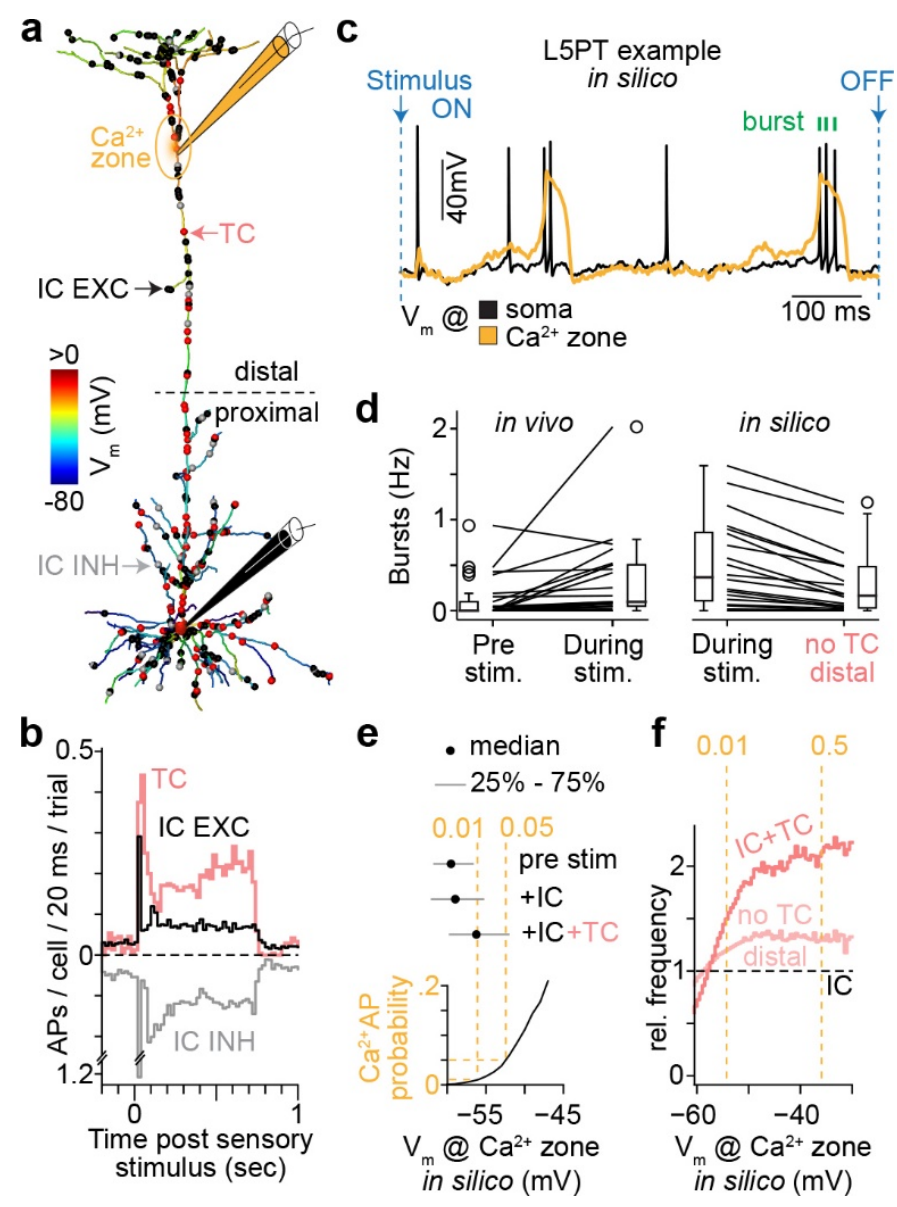

Fig. 3: Thalamus gates the generation of calcium APs in cortical output neurons in silico. a. Example multi-scale model for the L5PT shown in Fig. 1b. The soma, dendrites and TC synapses of the in vivo recorded L5PT were converted into multi-compartmental models to capture the empirically measured cellular (Fig. S4a) and synaptic physiology (Fig. S4b) for this cell type (21). The cellular models were embedded into a circuit model for the intracortical (IC) connectivity in rat vS1 (Fig. S4c). The embedding provided estimates for the numbers, dendritic locations and cell type-specific cellular origins of IC excitatory (EXC) inputs, consistent with empirically measured connectivity data (15). Here we show $1 \%$ of IC and $5 \%$ of TC synapses for one example configuration of such anatomically constrained input patterns. b. PSTHs for EXC and inhibitory (INH) IC neurons, as well as for TC neurons, representing the data in Fig. 2 and S3. We activated the anatomically constrained inputs according to these cell type-specific activity recordings. c. We simulated how L5PTs integrate and transform such anatomically and functionally constrained TC and IC input patterns into AP output. Here we show the in silico predicted activity for one example input configuration during sensory stimulation. d. Burst rates across in vivo recorded L5PTs ( $\mathrm{n}=25$; left) versus in silico predicted burst rates across multi-scale models ( $\mathrm{n}=25$; right) with and without distal TC synapses. The in silico predicted burst rates during sensory stimulation match the in vivo recordings (two sided t-Test unpaired, $\mathrm{p}=0.40$ ). Without distal TC input, the simulations predict a significant reduction of bursts (two-sided t-Test paired, $\mathrm{p}<10^{-5}$ ). e. We measured the membrane potential at the initiation zone for calcium $\left(\mathrm{Ca}^{2+}\right)$ APs in simulation trials without a sensory stimulus (pre stim), and with a sensory stimulus without $(+\mathrm{IC})$ and with TC input $(+\mathrm{IC}+\mathrm{TC})$. Lower panel: probability to observe $\mathrm{Ca}^{2+}$ APs as a function of the membrane potential. f. Distal, but not proximal, TC inputs facilitate the generation of $\mathrm{Ca}^{2+} \mathrm{APs}$. 
Could the wiring specificity in the TC $\rightarrow$ L5PT pathway underlie this characteristic increase of burst firing in the major cortical output neurons? We performed cell-attached recordings in VPM thalamus, which showed that TC neurons $(n=8)$ respond reliably with APs for the duration of the sensory stimulus (Fig. 2c). Thus, direct TC input could in principle contribute to the increased burst firing of L5PTs under these experimental conditions. However, disentangling the impact of direct TC inputs from those that L5PTs receive from IC populations that are directly (or indirectly) driven by the thalamus represents a major challenge. To address this challenge, we generated biophysically detailed multi-compartmental models from the reconstructed L5PTs (Fig. S4a), converted the TC synapses along their dendrites into conductance-based models, and adjusted their respective strengths (Fig. S4b) to match with empirical data (10). We embedded these synaptic and cellular models into a circuit model of the barrel cortex $(13,15)$, which provided anatomically realistic constraints for which IC neurons provide input to the L5PT models (Fig. S4c), and where along their dendrites (Fig. 3a). Finally, we used our recordings for the different cell types in barrel cortex and VPM thalamus to constrain which of the anatomically possible connections provide functional input to the L5PT models - before and during the stimulus (Fig. 3b). This multi-scale model design allowed us simulate how well-constrained TC and IC input patterns are actively integrated by the L5PT dendrites and transformed into sensory-evoked cortical output patterns (Fig. 3c).

Without tuning the parameters at synaptic, cellular, or circuit scales, the simulations produced cortical output patterns that were remarkably consistent with our recordings (Fig. S4d). The simulations captured the fast onset latencies of the sensory-evoked AP responses, as well as the firing and burst rates of L5PTs during the stimulus (Fig. 3d). Surprisingly, when we deprived the L5PT models of distal direct TC input, the sensory-evoked cortical output patterns appeared largely unchanged (Fig. S4e). However, sensoryevoked bursts were significantly (two-sided t-Test paired: $\mathrm{p}<10^{-4}, \mathrm{n}=25$ L5PT models) reduced (Fig. 3d). Therefore, we systematically measured the membrane potential around the dendritic calcium domain during simulations with and without distal TC inputs. The median difference between these two in silico scenarios was $1.6 \mathrm{mV}$. Despite being small, this extra depolarization by distal TC inputs persistently advanced the membrane potential close to the threshold for calcium APs (Fig. 3e). Distal inputs from IC sources thereby appeared amplified at the cell body (Fig. S4e), and had higher probabilities to elicit calcium APs (Fig. 3f). These simulation results indicate that distal TC input to L5PTs is generally not sufficient to drive calcium APs, yet it facilitates their generation during sensory processing. Consistent with the in silico predictions are in vitro recordings, which reported oppositely that injecting a current that hyperpolarizes the membrane by $\sim 1 \mathrm{mV}$ near the primary branch point is sufficient to abolish calcium APs in L5PTs (5). Thus, wiring specificity in the TC $\rightarrow$ L5PT pathway is predicted to function as gating mechanism for the generation of calcium APs. Specifically, TC gating ensures that distal dendritic inputs can impact cortical output patterns by generating bursts of axonal APs during sensory processing.

How is it possible to test the TC gating mechanism empirically? The simulations indicate that L5PTs require distal inputs from both TC and IC sources to generate calcium APs. Thus, L5PTs should transition in vivo from single AP to burst firing with increasing amounts of IC input that is provided at the time of direct TC input. We were able to quantitatively test this prediction, because of an unexpected observation that we made when optogenetically activating the TC synapses (Methods). As could be expected from in vitro studies $(22,23)$, neurons across layers and cell types (58 out of 71$)$ responded with short latency APs during $10 \mathrm{~ms}$ long light pulses that were provided every $2.5 \mathrm{sec}$ onto the surface of the barrel cortex (Fig. S5a). All L5PTs responded reliably with typically a single AP, rarely with two APs (19\% probability across all L5PTs), but almost never (only in 1 out of 24 L5PTs) with bursts of three or more APs - an indication that TC input is generally not sufficient to drive calcium APs (5). Unexpectedly, however, we 
observed that the barrel cortex remained in an excited stated for up to $600 \mathrm{~ms}$ after each light stimulus (Fig. 4a). We quantified this phenomenon by measuring the local field potential (LFP) prior and during light stimulation (Fig. 4b). A prolonged depolarization of the LFP was consistently observed across layers, indicating that the entire local network remains excited for an extended period of time. We therefore considered if we stimulated the TC synapses again with light while the network is still excited, would the additional depolarization by IC sources be sufficient to cause the predicted transition from single AP to burst firing in L5PTs? For this purpose, we decreased the intervals between light stimuli to $100 \mathrm{~ms}$. In a subset of experiments, this stimulation resulted in an excited state that persisted throughout all trials (Fig. 4c). It was in these experiments that we observed L5PTs to switch to burst firing (Fig. 4d). The more depolarized the LFP was at the onset of the stimulus, the higher was the probability to evoke bursts in L5PTs (Fig. 4e, S5b). This IC state-dependent transition was not observed in other cell types (Fig. S5b/c). Thus, consistent with the in silico predicted gating function, we found that the amount of IC excitation at the time of TC input determines whether cortical output patterns transition from single AP to burst firing - a phenomenon which is due to the wiring specify in the TC $\rightarrow$ L5PT pathway.

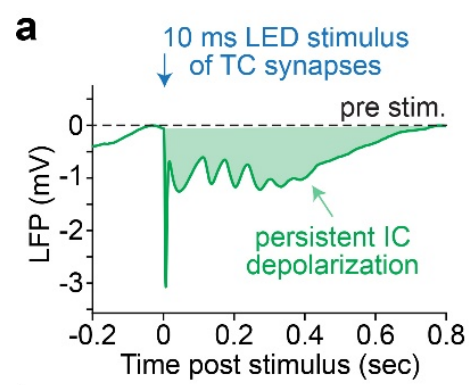

b
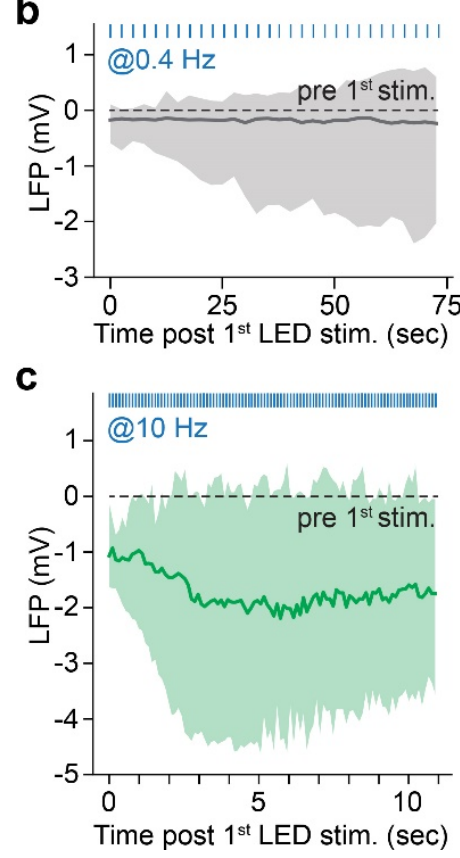

d

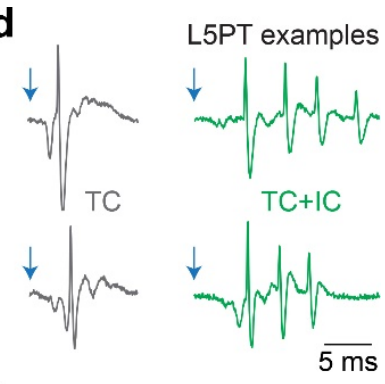

e

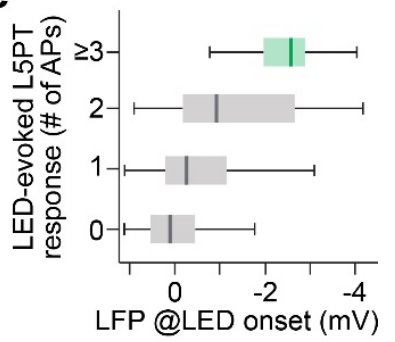

$\mathbf{f}$

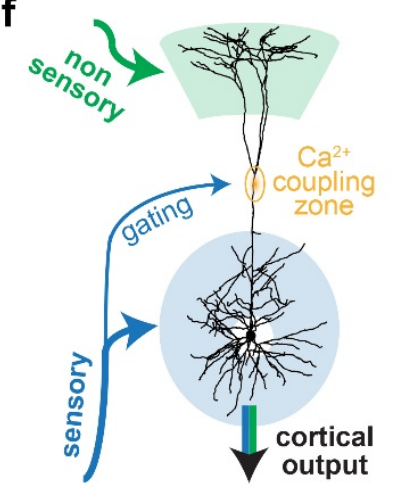

Fig. 4: Thalamus gates the generation of calcium APs in cortical output neurons in vivo. a. Example recording of local field potential (LFP) in vS1 after activation of TC synapses by a $10 \mathrm{~ms}$ pulse of light. b. LFP recording throughout stimulation with $10 \mathrm{~ms}$ pulses at $2.5 \mathrm{sec}$ interstimulus intervals. Average (grey line) and standard deviation (STD, shaded area) across 17 rats. c. Same as in panel b but for $100 \mathrm{~ms}$ interstimulus intervals, and for those recordings in which we observed burst responses in L5PTs $(n=6)$. d. Two example L5PTs that showed the transition from single APs (@0.4 Hz) to bursts of $\geq 3$ APs (@10 Hz). e. The higher the IC depolarization (i.e., LFP) at the onset of the light stimulus, the higher the probability that TC input evoked bursts. f. Proposed circuit theme by which L5PTs combine sensory with non-sensory information into cortical output. Primary thalamus directly and indirectly (i.e., via L4 (24) and L6CC (13)) provides sensory information to the proximal dendrites of L5PTs, and directly gates active dendritic computations to integrate this information with distal inputs from top-down pathways $(22,25)$.

\section{Discussion}

In this study we sought to understand why the primary thalamus relays sensory information directly to the major cortical output neurons. In contrast to the conventional view which assumes that direct TC input to neurons in layers $5 / 6$ is modulatory $(8,26-28)$, it was proposed that thalamus might directly drive two 
separate processing systems in parallel: an upper stratum (TC $\rightarrow \mathrm{L} 4 \rightarrow \mathrm{L} 2 / 3)$ and a lower stratum $(\mathrm{TC} \rightarrow \mathrm{L} 5 / 6)(10)$. In support of a bistratified cortical system, we had recently reported that a subpopulation of corticocortical neurons (L6CCs) clusters around the innervation domain of TC axons at the layer 5 to 6 border (13). These L6CCs reliably relay sensory-evoked TC input to the proximal dendrites of L5PTs. Inactivating L6CCs abolishes sensory-evoked APs in L5PTs. Thus, input from L6CCs is necessary for L5PTs to transform proximal inputs during sensory processing into AP responses. Consequently, it is indeed a lower stratum $(\mathrm{TC} \rightarrow \mathrm{L} 6 \mathrm{CC} \rightarrow \mathrm{L} 5 \mathrm{PT})$ which accounts for the fast onsets of sensory-evoked cortical output patterns - an organizing principle that is likely to generalize across sensory systems and species (29-33). However, direct input from the thalamus is generally not sufficient to drive sensory-evoked APs in L5PTs (13). Instead, here we found that TC axons specifically and densely target the calcium domain in the distal dendrites of L5PTs. By complementing this anatomical observation with electrophysiological, computational and optogenetic manipulation evidence - acquired consistently for the same in vivo recorded neurons - we show that the striking wiring specificity in the TC $\rightarrow$ L5PT pathway gates the generation of calcium APs during sensory processing. Thus, primary thalamus implements three parallel functions that complement one another. TC input to layer 4 activates cortical sensory processing (24). TC input to the layer $5 / 6$ border activates a lower stratum, which enables L5PTs to transform proximal inputs (e.g. from the canonical circuit (8)) into cortical output patterns (13). TC input to L5PTs activates an active dendritic mechanism, which enables distal inputs to modulate cortical output patterns with bursts of APs. Taken together, our findings indicate that direct sensory input to the major cortical output neurons facilitates that the results of sensory processing from a bistratified cortical system are reliably combined with information streams from top-down pathways that terminate in layer $1(1,3)$ (Fig. 4f).

Could TC gating in the distal dendrites of L5PTs contribute to their role during perception? Several observations support such an interpretation of our data. First, neurons in layers $2 / 3$ of the barrel cortex, which project either to the secondary somatosensory (S2) or primary motor (M1) cortex, are selectively activated depending on the whisker-mediated behavioral task (34). Neurons in both S2 and M1 project long-range axons to layer 1 and layers 5/6 of the barrel cortex (25), where they can form synaptic connections with L5PTs (22). Contextual information is therefore likely to impinge onto the proximal and distal dendrites of L5PTs in the barrel cortex via stimulus induced parallel activation of these corticocortical feedback pathways. Thus, TC gating could facilitate the combination of sensory with contextual information - a process that is thought to form a basis for the L5PTs' role during perception (2). Second, in vitro recordings suggested that back-propagation of APs into the apical dendrite could provide the necessary depolarization for distal inputs to evoke calcium APs (5). However, backpropagating APs have little impact on the apical dendritic calcium signals observed in vivo (35, 36). The calcium signals in L5PTs that correlate with the perceptual threshold were hence interpreted to represent local activation of calcium channels rather than back-propagating APs (6). Here we showed that direct TC input specifically and persistently depolarizes the dendritic calcium domain of L5PTs during sensory stimulation, which is consistent with the interpretation of local activation. Finally, calcium signals that correlate with perception were only observed in L5PTs, but not in other layer 5 projection neurons (2). Providing a likely explanation for this observation, we found that the wiring specificity in the TC $\rightarrow$ L5PT pathway was not apparent for other deep layer cell types. Taken together, we therefore propose that by targeting the major cortical output neurons directly, primary thalamus facilitates that the sensory information it relays to cortex can be perceived by the animal.

Our data revealed that long-range axons are able to target an active dendritic mechanism in a specific cell type, even if the location of the dendritic target domain varies from cell-to-cell. Recently, it was suggested 
that such an extreme level of wiring specificity might be necessary for cortical circuits to implement higher brain functions - and potentially even conscious processing (1). However, it had yet to be demonstrated that such wiring specificity exists. Thus, our findings might represent a general circuit theme for how calcium APs are utilized to associate inputs from anatomically segregated information streams.

\section{Materials and Methods}

Virus injection: Male Wistar rats were provided by Charles River Laboratories. All experiments were carried out after evaluation by the local German authorities, and in accordance with the animal welfare guidelines of the Max Planck Society. Primary thalamic axons/boutons were virus labeled as described previously (13). Briefly, rats aged 22-25 days (P22-25) were anesthetized with isoflurane supplemented by Caprofen $(5 \mathrm{mg} / \mathrm{kg})$ and Buprenorphine SR $(1 \mathrm{mg} / \mathrm{kg})$ as analgesia, then placed into a stereotaxic frame (Kopf Instruments, model 1900), and provided with a continuous flow of isoflurane/O2 gas. Body temperature was maintained at $37^{\circ} \mathrm{C}$ by a heating pad. A small craniotomy was made above the left hemisphere $2.85 \mathrm{~mm}$ posterior to bregma and $3.2 \mathrm{~mm}$ lateral from the midline. The head of the rat was leveled with a precision of $1 \mu \mathrm{m}$ in both the medial-lateral and anterior-posterior planes using an eLeVeLeR electronic leveling device (Sigmann Electronics, Hüffenhardt, Germany) mounted to an adaptor of the stereotaxic frame. An injecting pipette containing an adeno-associated virus (AAV) was lowered into the VPM thalamus (5.05 mm from the pia). The virus (37) - rAAV2/1-Syn-hChR2(H134R)mCherry (titer: $1 \times 10^{12} \mathrm{gc} \mathrm{ml}^{-1}$ ) - was provided by Martin Schwarz (University of Bonn, Germany). 50-70 $\mathrm{nL}$ of the virus were injected using a 30cc syringe coupled to a calibrated glass injection capillary.

Electrophysiology: Non-virus injected rats (P28-48), as well as AAV injected rats (after a 16-21 day incubation period) were anesthetized with urethane $(1.8 \mathrm{~g} / \mathrm{kg}$ body weight $)$ by intraperitoneal injection. The depth of anesthesia was assessed by monitoring pinch withdrawal, eyelid reflexes, and vibrissae movements. Body temperature was maintained at $37.5 \pm 0.5^{\circ} \mathrm{C}$ by a heating pad. Cell-attached recording and biocytin labeling was performed as described in detail previously (38). Briefly, a small craniotomy was made above the left hemisphere $2.5 \mathrm{~mm}$ posterior and $5.5 \mathrm{~mm}$ lateral to the bregma (for recordings in vS1), or 2.9-3.5 mm anterior to the bregma, 2.4-3.4 mm lateral from the midline, and at 5-6 mm depth from the pia (for recordings in VPM thalamus). APs were recorded using an extracellular loose patch amplifier (ELC-01X, npi electronic $\mathrm{GmbH}$ ) or an Axoclamp 2B amplifier (Axon instruments, Union City, CA, USA), digitized using a CED power1401 data acquisition board (CED, Cambridge Electronic Design, Cambridge, UK), and low pass filtered $(300 \mathrm{~Hz})$ to measure the LFP. APs were recorded before and during 20-30 trials of caudal multi-whisker deflections by a $700 \mathrm{~ms}$ airpuff (10 PSI), delivered through a $1 \mathrm{~mm}$ plastic tube from a distance of $8-10 \mathrm{~cm}$ from the whisker pad (39). Stimulation was repeated at constant intervals $(0.3 \mathrm{~Hz})$. In AAV injected rats, optical stimulation of ChR2-expressing thalamocortical terminals was provided by a $400 \mu \mathrm{m}$ diameter optical fiber (ThorLabs \#RJPSF2) coupled to a $470 \mathrm{~nm}$ wavelength LED source (ThorLabs M470F3) and powered by an LED driver (ThorLabs \#DC2200). A 10 ms pulse of light generated $1 \mathrm{~mW}$ output power at the end of the optical fiber as measured by a laser power meter (ThorLabs \#PM100A) coupled to a photodiode (ThorLabs \#S121C). The optical fiber was positioned using a 3-axis motorized micromanipulator (Luigs and Neuman) approximately 1-2 $\mathrm{mm}$ above the cortical surface, so that the light beam resulted in a 1-2 mm disc of light above the recording site in vS1. Control of the LED driver was implemented with Spike2 software (CED, Cambridge, UK.). APs were recorded during 20-100 trials of $10 \mathrm{~ms}$ light pulses, at inter-stimulus intervals of $2.5 \mathrm{sec}$ or $100 \mathrm{~ms}$. Following the electrophysiological measurements, neurons were labeled with biocytin. Labeling sessions were repeated several times. After 1-2 hours for biocytin diffusion, animals were transcardially perfused with $0.1 \mathrm{M}$ 
phosphate buffer (PB) followed by 4\% paraformaldehyde (PFA). Brains were removed and post-fixed with $4 \%$ PFA for 8 to 12 hours, transferred to $0.1 \mathrm{M}$ PB and stored at $4{ }^{\circ} \mathrm{C}$.

Histology: For recordings in vS1 of non-virus injected rats, 22-25 consecutive $100 \mu \mathrm{m}$ thick vibratome sections were cut tangentially to $\mathrm{vS} 1\left(45^{\circ}\right.$ angle) ranging from the pial surface to the white matter, or coronally (for recordings in VPM thalamus). All sections were treated with avidin-biotin (ABC) solution, and subsequently neurons were identified using the chromogen 3,3'-diaminobenzidine tetrahydrochloride (DAB). All sections were mounted on glass slides, embedded with Mowiol, and enclosed with a cover slip. For recordings in AAV injected rats, the recorded hemisphere was cut tangentially into 45-48 consecutive $50 \mu \mathrm{m}$ thick vibratome sections from the pial surface to the white matter. The remaining brain tissue was embedded in 10\% gelatin (Sigma Aldrich \#G2500) and cut coronally into consecutive $50 \mu \mathrm{m}$ thick sections to identiy the virus injection site. Sections were first washed three times in $0.1 \mathrm{M}$ PB and treated with streptavidin conjugated to AlexaFluor488 $(5 \mu \mathrm{g} / \mathrm{ml})$ (Molecular Probes \#S11223) in 0.1 M PB containing 0.3\% Triton X-100 (TX) (Sigma Aldrich \#9002-93-1), $400 \mu$ l per section for 3-5 hours at room temperature in order to visualize biocytin labeled neuronal structures. To enhance the fluorescence expressed by the virus and to label primary thalamic synapses, slices were then double immunolabeled with anti-mCherry antibody and anti-VGlut2 antibody. Sections were permeabilized and blocked in $0.5 \%$ Triton x-100 (TX) (Sigma Aldrich \#9002-93-1) in 100 mM PB containing 4\% normal goat serum (NGS) (Jackson ImmunoResearch Laboratories \#005-000-121) for 2 hours at room temperature. The primary antibodies were diluted 1:500 (Rabbit anti-mCherry, Invitrogen \#PA5-34974 Invitrogen \#M11217 and mouse anti-VGlut2 antibody, Synaptic Systems \#135421) in PB containing 1\% NGS for 48 hours at $4^{\circ} \mathrm{C}$. The secondary antibodies were diluted 1:500 (goat anti-Rabbit IgG Alexa-647 H+L Invitrogen \#A21245 and goat anti-Mouse IgG Alexa-405 H+L Invitrogen \#A31553), and were incubated for 2-3 hours at room temperature in PB containing 3\% NGS and 0.3\% TX. All sections were mounted on glass slides, embedded with SlowFade Gold (Invitrogen \#S36936) and enclosed with a coverslip.

Morphological reconstructions: Neuronal structures were extracted from image stacks using a previously reported automated tracing software (40). For reconstruction of fluorescently labeled neurons and locating of AAV labeled TC synapses, images were acquired using a confocal laser scanning system (Leica Application Suite Advanced Fluorescence SP5; Leica Microsystems). 3D image stacks of up to $2.5 \mathrm{~mm} \times$ $2.5 \mathrm{~mm} \times 0.05 \mathrm{~mm}$ were acquired at a resolution of $0.092 \times 0.092 \times 0.5 \mu \mathrm{m}$ per voxel $(63 \mathrm{x}$ magnification, NA 1.3). Image stacks were acquired for each of 45-48 consecutive $50 \mu \mathrm{m}$ thick tangential brain slices that range from the pial surface to the white matter. Manual proof-editing of individual sections, and automated alignment across sections were performed using custom-designed software (41). Pia, barrel and white matter outlines were manually drawn on low-resolution images (4x magnification dry objective). Using these anatomical reference structures, all reconstructed morphologies were registered to a standardized 3D reference frame of rat vS1 (42). The shortest distance from the pial surface to the soma, and 20 morphological features that have previously been shown to separate between excitatory cell types in rat vS1 (43) were calculated for each registered morphology. For identification of putative TC synapses, biocytin labeled morphologies and AAV labeled VPM terminals were imaged simultaneously using the confocal laser scanning system as described above: biocytin Alexa-488 (excited at $488 \mathrm{~nm}$, emission detection range 495-550 nm), AAV Alexa-647 (excited at $633 \mathrm{~nm}$, emission detection range 650-785 nm). These dual-channel image stacks were loaded into Amira software (Thermo Scientific) for visualization. All reconstructed dendrites were manually inspected, and landmarks were placed onto each spine head if a spine head was overlapping with a VPM bouton, to mark the location of a putative synapse. The shortest distance of each landmark to the dendrite reconstruction was determined, and the path length distance was 
calculated from that location along the reconstructed neuron to the soma. For validation of putative TC synapses, image stacks were acquired with an up-right super-resolution enhanced confocal laser scanning system (Leica Application Suite Advance Lightening Fluorescence SP8; Leica Microsystems) via a glycerol/oil immersion objective (HCX PL APO 63x magnification, NA 1.3), a tandem scanning system ( $8 \mathrm{kHz}$ resonance scanning speed), and spectral detectors with hybrid technology (GaAsP photocathode; 8x line average): VGlut2 Alexa-405 (excited at $405 \mathrm{~nm}$, emission detection range: 410-480 nm), biocytin Alexa-488 (excited at $488 \mathrm{~nm}$, emission detection range 495-550 nm), AAV Alexa-647 (excited at 633 $\mathrm{nm}$, emission detection range $650-785 \mathrm{~nm}$ ). Triple-channel image stacks were acquired at a resolution of $29.5 \times 29.5 \times 130 \mathrm{~nm}$ per voxel. Image stacks were visualized in Amira, and manually inspected for overlap within a single optical section between spine heads and AAV- and/or VGlut2-positive boutons.

Multi-compartmental models: Four multi-compartmental models were used in this study. The models were generated as previously described $(13,21)$. Briefly, a simplified axon morphology was attached to the soma of the L5PT morphology from Fig. 1b (44). The axon consisted of an axon hillock with a diameter tapering from $3 \mu \mathrm{m}$ to $1.75 \mu \mathrm{m}$ over a length of $20 \mu \mathrm{m}$, an axon initial segment of $30 \mu \mathrm{m}$ length and diameter tapering from $1.75 \mu \mathrm{m}$ to $1 \mu \mathrm{m}$ diameter, and $1 \mathrm{~mm}$ of myelinated axon (diameter of $1 \mu \mathrm{m}$ ). Next, a multi-objective evolutionary algorithm was used to tune the passive leak conductance and the density of Hodgkin-Huxley type ion channels on soma, basal dendrite, apical dendrite and axon initial segment, such that the neuron model is able to reproduce characteristic electrophysiological responses to somatic and dendritic current injections of L5PTs within the experimentally observed variability, including back-propagating APs, calcium APs, and AP responses to prolonged somatic current injections (21). We augmented the original biophysical model of L5PTs $(13,21)$ with two ion channel parameters: according to a previous report (45), the density of the fast non-inactivating potassium channels (Kv3.1) was allowed to linearly decrease with soma distance until it reaches a minimum density (i.e., the slope and minimum density are two additional parameters). We incorporated the IBEA algorithm (44) for optimization. The optimization was terminated if there was no progress or when acceptable models had been found. We repeated the optimization process several times and could generate acceptable models in 4 independent runs. From each run, we selected one model for which the maximal deviation from the experimental mean in units of standard deviation across all objectives was minimal.

Connectivity models: TC synapse positions along the L5PT models were measured empirically as described above. Cell type-specific IC excitatory connections are derived from an anatomically realistic circuit model of rat vS1 (15), a procedure, which has been described in detail previously (13). We placed the dendrite morphology selected for multi-compartmental modelling in the circuit model at nine locations within the cortical barrel column representing the $\mathrm{C} 2$ whisker, which is located approximately in the center of vS1, while preserving its (in vivo) soma depth. The locations were the column center, and equally spaced angular intervals with a distance of $\sim 100 \mu \mathrm{m}$ to the column center. For each of the nine locations, we estimated the numbers and dendritic locations of cell type-specific synapses that impinge onto the dendrites of the L5PT model. Finally, IC inhibitory synapses were distributed randomly on the dendritic tree. Synapses were assigned to presynaptic neurons in the following manner. TC synapses: we randomly assigned each TC synapse to $350 \mathrm{TC}$ neurons, which is the average number of neurons in the somatotopically aligned C2 barreloid (46). IC excitatory synapses: presynaptic neuron IDs are an output of the circuit model (15). IC inhibitory synapses: each synapse was assigned to one virtual neuron.

Synapse models: Synapse models and synaptic parameters (rise and decay times, release probabilities, reversal potentials) were reported previously (13). Briefly, conductance-based synapses were modeled 
with a double-exponential time course. Excitatory synapses contained both AMPA receptors (AMPARs) and NMDARs. Inhibitory synapses contained $\mathrm{GABA}_{\mathrm{A}} \mathrm{Rs}$. The peak conductance of excitatory synapses from different presynaptic cell types was determined by assigning the same peak conductance to all synapses of the same cell type, activating all connections of the same cell type (i.e., all synapses originating from the same presynaptic neurons) one at a time, and comparing parameters of the resulting unitary postsynaptic potential (uPSP) amplitude distribution (mean, median and maximum) for a fixed peak conductance with experimental measurements in vitro (IC input (47)) or in vivo (TC input (10), Fig. S4b). The peak conductance for synaptic inputs from each cell type was systematically varied until the squared differences between parameters of the in silico and in vitro/in vivo uPSP amplitude distributions were minimized (i.e., the mean, median and maximum of the distributions were used, and mean and median were weighted twice relative to the maximum). This procedure was repeated for each of the four multi compartmental models using the connectivity model for the location in the center of the $\mathrm{C} 2$ column (Table S1). The peak conductance at inhibitory synapses was fixed at $1 \mathrm{nS}(48)$.

Simulations: Simulations were performed using Python 2.7 and NEURON 7.4 (49). Synaptic activity representing $700 \mathrm{~ms}$ air puff stimulation was generated by activating the presynaptic neurons determined by the connectivity model with empirically observed cell type-specific activity (Fig. S3). From our recording data, we computed the cell type-specific firing frequencies before stimulation (-700 $\mathrm{ms}$ to $0 \mathrm{~ms})$, during the onset response (characterized by a sharp peak of activity, 20 to $40 \mathrm{~ms}$ post stimulus), and during the remaining duration of the stimulus (40 to $700 \mathrm{~ms}$ ). We then generated Poisson spike trains for each presynaptic neuron based on these measurements (using the ongoing frequency up to the time point of 20 ms post stimulus onset). We tuned the spontaneous and onset frequency of inhibitory neurons such that the L5PT models produce spontaneous firing rates within the range we observed empirically $(0.1-9.5 \mathrm{~Hz})$, and generate single APs or doublets of APs but no bursts of three APs during the onset period, consistent with our empirical data. To ensure the simulation results are independent of the particular level of inhibition the L5PT models receive, we systematically varied the sustained frequency of inhibitory neurons by a factor ranging from 0.5 to 3 ( 0.1 increment from 0.5 to 1.2 and 0.2 above that, resulting in 17 different values). At the lower and higher end of this range, spike rates were either excessively high or completely suppressed. We found spiking frequencies in the empirically observed range $(0.3$ to $22.9 \mathrm{~Hz})$ for five factors $(0.9$ to 1.4$)$. The simulations thereby comprise 4 multi-compartmental models, 9 connectivity models and 5 levels of inhibition, i.e., 180 configurations. In trials, where distal TC synapses were silenced, we removed TC synapses whose path length from the soma exceeded $500 \mu \mathrm{m}$. To investigate the influence of distal IC synapses on the soma (Fig. S4e), we re-simulated the 180 configurations with identical synaptic input (both in control conditions and without distal TC synapses), but activated one additional excitatory synapse with a maximum conductance of $1 \mathrm{nS}$ at a randomly selected time point $(40-700 \mathrm{~ms})$, and on a randomly selected segment of the apical tuft. We determined the time point of calcium APs as the time point of up-crossing a threshold of $-30 \mathrm{mV}$ of the membrane potential which was recorded at the primary branch point of the apical dendrite - i.e., the center of the dendritic calcium zone (for recording position see Fig. 3a). To determine the threshold, the membrane potential was plotted against its first derivative. The threshold was determined as the characteristic point beyond which the membrane potential always has a positive slope. The threshold was similar for the 4 biophysical models investigated. To analyze the distribution of the membrane potential, we preprocessed the simulation data to remove suprathreshold events. We aligned all voltage traces by the time point of calcium APs. We determined the first time point at which further calcium APs can occur (i.e., the refractory period of the calcium zone), which ranged from 125 to $310 \mathrm{~ms}$, depending on the multicompartmental model. Finally, the voltage trace intervals between the calcium AP up to the refractory 
period where discarded. From the remaining values, we computed the median and $25 / 75 \%$ percentiles across all simulation trials at time points from 40-700 ms post stimulus. We computed the calcium AP probability as the probability to encounter it within $20 \mathrm{~ms}$ as a function of the membrane potential at the calcium zone. To analyze the influence of distal synapses on the somatic subthreshold potential, we computed the absolute difference of the somatic membrane potential between the original and the resimulated data in which one additional synapse had been activated. We discarded time periods in which suprathreshold events were occurring, and computed the integral over the remaining time intervals (unit: $\left.\mathrm{mV}^{*} \mathrm{~ms}\right)$. Bursts were defined as the occurrence of three APs within 30ms. The model and simulation routines, including a documentation of all parameters and the analysis routines can be obtained from ModelDB (https://senselab.med.yale.edu/ModelDB/; accession number: 267166).

Quantification and statistical analysis: All data are reported as mean \pm standard deviation (STD) unless mentioned otherwise. All of the statistical details can be found in the figure legends, figures, and Results, including the statistical tests used, sample size n, and what the sample size represents (e.g. number of animals, number of cells). Significance was defined for $\mathrm{p}$-values smaller than 0.05 . Boxplots show median, $25 \%$ and $75 \%$ percentile; whiskers extend up to 1.5 times the interquartile range. All data beyond the whiskers are shown as outliers. All tests were performed using the scipy python package (version 0.18.2).

\section{References}

1. J. Aru, M. Suzuki, M. E. Larkum, Cellular Mechanisms of Conscious Processing. Trends Cogn Sci 24, 814-825 (2020).

2. N. Takahashi et al., Active dendritic currents gate descending cortical outputs in perception. Nat Neurosci 23, 1277-1285 (2020).

3. K. D. Harris, G. M. Shepherd, The neocortical circuit: themes and variations. Nat Neurosci 18, 170$181(2015)$.

4. J. Schiller, Y. Schiller, G. Stuart, B. Sakmann, Calcium action potentials restricted to distal apical dendrites of rat neocortical pyramidal neurons. J Physiol 505 ( Pt 3), 605-616 (1997).

5. M. E. Larkum, J. J. Zhu, B. Sakmann, A new cellular mechanism for coupling inputs arriving at different cortical layers. Nature 398, 338-341 (1999).

6. N. Takahashi, T. G. Oertner, P. Hegemann, M. E. Larkum, Active cortical dendrites modulate perception. Science 354, 1587-1590 (2016).

7. R. J. Douglas, K. A. Martin, Neuronal circuits of the neocortex. Annu Rev Neurosci 27, 419-451 (2004).

8. R. J. Douglas, K. A. Martin, in Handbook of Brain Microcircuits, G. M. Shepherd, S. Grillner, Eds. (Oxford University Press, 2010).

9. C. D. Gilbert, T. N. Wiesel, Morphology and intracortical projections of functionally characterised neurones in the cat visual cortex. Nature 280, 120-125 (1979).

10. C. M. Constantinople, R. M. Bruno, Deep cortical layers are activated directly by thalamus. Science 340, 1591-1594 (2013).

11. M. Armstrong-James, K. Fox, Spatiotemporal convergence and divergence in the rat S1 "barrel" cortex. J Comp Neurol 263, 265-281 (1987).

12. C. P. de Kock, R. M. Bruno, H. Spors, B. Sakmann, Layer- and cell-type-specific suprathreshold stimulus representation in rat primary somatosensory cortex. J Physiol 581, 139-154 (2007).

13. R. Egger et al., Cortical Output Is Gated by Horizontally Projecting Neurons in the Deep Layers. Neuron 105, 122-137 e128 (2020). 
14. M. Ito, Simultaneous visualization of cortical barrels and horseradish peroxidase-injected layer $5 \mathrm{~b}$ vibrissa neurones in the rat. J Physiol 454, 247-265 (1992).

15. D. Udvary et al., The Impact of Neuron Morphology on Cortical Network Architecture. 2021 (https://www.biorxiv.org/content/10.1101/2020.11.13.381087v4).

16. H. G. Kim, B. W. Connors, Apical dendrites of the neocortex: correlation between sodium- and calcium-dependent spiking and pyramidal cell morphology. J Neurosci 13, 5301-5311 (1993).

17. J. Magee, D. Hoffman, C. Colbert, D. Johnston, Electrical and calcium signaling in dendrites of hippocampal pyramidal neurons. Annu Rev Physiol 60, 327-346 (1998).

18. W. A. Spencer, E. R. Kandel, Electrophysiology of Hippocampal Neurons: Iv. Fast Prepotentials. $J$ Neurophysiol 24, 272-285 (1961).

19. R. Yuste, M. J. Gutnick, D. Saar, K. R. Delaney, D. W. Tank, Ca2+ accumulations in dendrites of neocortical pyramidal neurons: an apical band and evidence for two functional compartments. Neuron 13, 23-43 (1994).

20. C. P. De Kock et al., High-frequency burst spiking in layer 5 thick-tufted pyramids of rat primary somatosensory cortex encodes exploratory touch. Communications Biology, (2021).

21. E. Hay, S. Hill, F. Schurmann, H. Markram, I. Segev, Models of neocortical layer 5b pyramidal cells capturing a wide range of dendritic and perisomatic active properties. PLoS Comput Biol 7, e1002107 (2011).

22. L. Petreanu, T. Mao, S. M. Sternson, K. Svoboda, The subcellular organization of neocortical excitatory connections. Nature 457, 1142-1145 (2009).

23. B. S. Sermet et al., Pathway-, layer- and cell-type-specific thalamic input to mouse barrel cortex. Elife 8, (2019).

24. R. M. Bruno, B. Sakmann, Cortex is driven by weak but synchronously active thalamocortical synapses. Science 312, 1622-1627 (2006).

25. T. Mao et al., Long-range neuronal circuits underlying the interaction between sensory and motor cortex. Neuron 72, 111-123 (2011).

26. E. M. Callaway, Feedforward, feedback and inhibitory connections in primate visual cortex. Neural Netw 17, 625-632 (2004).

27. R. W. Guillery, S. M. Sherman, Thalamic relay functions and their role in corticocortical communication: generalizations from the visual system. Neuron 33, 163-175 (2002).

28. A. N. Viaene, I. Petrof, S. M. Sherman, Synaptic properties of thalamic input to the subgranular layers of primary somatosensory and auditory cortices in the mouse. J Neurosci 31, 12738-12747 (2011).

29. A. Antonini, M. P. Stryker, Rapid remodeling of axonal arbors in the visual cortex. Science 260, 1819-1821 (1993).

30. F. Briggs, Organizing principles of cortical layer 6. Front Neural Circuits 4, 3 (2010).

31. F. Karube, K. Sari, Z. F. Kisvarday, Axon topography of layer 6 spiny cells to orientation map in the primary visual cortex of the cat (area 18). Brain Struct Funct 222, 1401-1426 (2017).

32. J. S. Lund, Anatomical organization of macaque monkey striate visual cortex. Annu Rev Neurosci 11, 253-288 (1988).

33. M. Velez-Fort et al., The stimulus selectivity and connectivity of layer six principal cells reveals cortical microcircuits underlying visual processing. Neuron 83, 1431-1443 (2014).

34. J. L. Chen, S. Carta, J. Soldado-Magraner, B. L. Schneider, F. Helmchen, Behaviour-dependent recruitment of long-range projection neurons in somatosensory cortex. Nature 499, 336-340 (2013).

35. M. Carandini, A. K. Churchland, Probing perceptual decisions in rodents. Nat Neurosci 16, 824-831 (2013). 
36. F. Helmchen, K. Svoboda, W. Denk, D. W. Tank, In vivo dendritic calcium dynamics in deep-layer cortical pyramidal neurons. Nat Neurosci 2, 989-996 (1999).

37. F. J. Meye et al., Shifted pallidal co-release of GABA and glutamate in habenula drives cocaine withdrawal and relapse. Nat Neurosci 19, 1019-1024 (2016).

38. R. T. Narayanan et al., Juxtasomal biocytin labeling to study the structure-function relationship of individual cortical neurons. J Vis Exp, e51359 (2014).

39. G. Rojas-Piloni et al., Relationships between structure, in vivo function and long-range axonal target of cortical pyramidal tract neurons. Nat Commun 8, 870 (2017).

40. M. Oberlaender, R. M. Bruno, B. Sakmann, P. J. Broser, Transmitted light brightfield mosaic microscopy for three-dimensional tracing of single neuron morphology. J Biomed Opt 12, 064029 (2007).

41. V. J. Dercksen, H. C. Hege, M. Oberlaender, The Filament Editor: an interactive software environment for visualization, proof-editing and analysis of 3D neuron morphology. Neuroinformatics 12, 325-339 (2014).

42. R. Egger, R. T. Narayanan, M. Helmstaedter, C. P. de Kock, M. Oberlaender, 3D reconstruction and standardization of the rat vibrissal cortex for precise registration of single neuron morphology. PLoS Comput Biol 8, e1002837 (2012).

43. M. Oberlaender et al., Cell type-specific three-dimensional structure of thalamocortical circuits in a column of rat vibrissal cortex. Cereb Cortex 22, 2375-2391 (2012).

44. E. Hay, F. Schurmann, H. Markram, I. Segev, Preserving axosomatic spiking features despite diverse dendritic morphology. J Neurophysiol 109, 2972-2981 (2013).

45. A. T. Schaefer et al., Dendritic voltage-gated K+ conductance gradient in pyramidal neurones of neocortical layer 5B from rats. $J$ Physiol 579, 737-752 (2007).

46. H. S. Meyer et al., Cellular organization of cortical barrel columns is whisker-specific. Proc Natl Acad Sci U S A 110, 19113-19118 (2013).

47. P. Schnepel, A. Kumar, M. Zohar, A. Aertsen, C. Boucsein, Physiology and Impact of Horizontal Connections in Rat Neocortex. Cereb Cortex 25, 3818-3835 (2015).

48. E. Hay, I. Segev, Dendritic Excitability and Gain Control in Recurrent Cortical Microcircuits. Cereb Cortex 25, 3561-3571 (2015).

49. M. L. Hines, N. T. Carnevale, The NEURON simulation environment. Neural Comput 9, 1179-1209 (1997).

50. R. T. Narayanan et al., Beyond Columnar Organization: Cell Type- and Target Layer-Specific Principles of Horizontal Axon Projection Patterns in Rat Vibrissal Cortex. Cereb Cortex 25, 44504468 (2015).

\section{Acknowledgments}

We thank Bert Sakmann for comments on the manuscript, and Martin Schwarz for providing the AAV. Funding was provided by the Center of Advanced European Studies and Research, the European Research Council under the European Union's Horizon 2020 research and innovation program (grant 633428, to M.O.), the Deutsche Forschungsgemeinschaft (SFB 1089 and SPP 2041, to M.O.), and the German Federal Ministry of Education and Research (grant 01IS18052, to M.O.). Author contributions: M.O. conceived and designed the study. J.G. performed experiments. A.B. developed the model and performed simulations. R.N. contributed data. J.G., A.B. and M.O. analyzed the data and wrote the paper. Declaration of interests: The authors declare no competing interests. 